\title{
Quand la concertation produit de l'évitement. La mise en œuvre de Natura 2000 en région Nord-Pas de Calais
}

\section{Agnès Fortier}

\section{(2) OpenEdition}

1 Journals

\section{Édition électronique}

URL : http://journals.openedition.org/developpementdurable/4602

DOI : 10.4000/developpementdurable.4602

ISSN : 1772-9971

Éditeur

Association DD\&T

\section{Référence électronique}

Agnès Fortier, «Quand la concertation produit de l'évitement. La mise en œuvre de Natura 2000 en région Nord-Pas de Calais », Développement durable et territoires [En ligne], Varia (2004-2010), mis en ligne le 28 juillet 2014, consulté le 10 décembre 2020. URL : http://journals.openedition.org/ developpementdurable/4602 ; DOI : https://doi.org/10.4000/developpementdurable.4602

Ce document a été généré automatiquement le 10 décembre 2020.

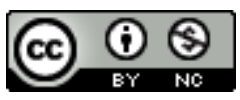

Développement Durable et Territoires est mis à disposition selon les termes de la licence Creative Commons Attribution - Pas d'Utilisation Commerciale 4.0 International. 


\title{
Quand la concertation produit de l'évitement. La mise en œuvre de Natura 2000 en région Nord-Pas de Calais
}

\author{
Agnès Fortier
}

1 La prise de conscience de l'impact des activités humaines sur la dynamique de la biosphère et $\mathrm{du}$ caractère limité des ressources naturelles ont conduit à faire de la préservation de la biodiversité une priorité à l'échelle planétaire (Barbault, Chevassus-auLouis, 2005). L'adoption en 1992, de la directive Habitats Faune-Flore répond à cette préoccupation à travers la création d'un réseau écologique européen baptisé Natura 2000. Cette politique, fondée sur des conceptions scientifiques nouvelles, entend ainsi concilier la conservation des habitats et des espèces reconnues d'importance communautaire avec la prise en compte des réalités culturelles, économiques et sociales, dans la perspective d'un développement durable. L'initiative prise dans ce cadre par le Ministère de l'Environnement français d'élaborer pour chaque site Natura 2000 un document d'objectifs (docob) assimilable à un plan de gestion relève d'une procédure concertée à l'échelle des territoires. Il s'agit pour l'ensemble des acteurs concernés de parvenir à un compromis sur la définition de normes de gestion compatibles avec la sauvegarde de la biodiversité. Le recours à telles procédures fondées sur la délibération et la concertation connaît un essor important dans le champ de l'action publique (Blondiaux, Sintomer, 2002 ; Mermet, 2004), en particulier dans le domaine de l'environnement et de la gestion des risques. La résolution de problèmes complexes, l'importance des controverses et la diversité des partenaires impliqués sont souvent invoquées pour justifier ce mode de conduite des affaires publiques. Délibérer est devenu en quelque sorte un passage obligé, un «impératif» (Blondiaux, Sintomer, 2002). Cependant, la mise en œuvre de ces modes d'action collectives ne produit pas toujours les effets escomptés. Les travaux entrepris par le réseau Grenat ${ }^{1}$ sur l'élaboration concertée des docob dans le cadre de la directive Habitats (DH), en France, ont permis de rendre compte de la variété des formes 
d'engagement des acteurs selon les sites. Le degré d'implication de ces derniers diffère notoirement selon l'importance des enjeux, l'état des rapports de force locaux ou encore selon le rôle joué par l'opérateur et le chargé de mission responsables de l'élaboration du docob. Si dans un certain nombre de cas de figure on constate une implication effective des partenaires tantôt sur le mode de la coopération, tantôt sur le mode du conflit, dans d'autres situations, on assiste à des formes de réticence de la part d'acteurs et d'institutions à s'engager dans le débat et la concertation. C'est précisément de cette situation dont nous souhaitons rendre compte ici, en examinant la procédure de coopération inter-établissements publics adoptée en région Nord-Pas de Calais (NPC). Audelà de son caractère inédit, cette expérience doit permettre de tirer un certain nombre d'enseignements sur les processus de délibération'2

2 Pour analyser les interactions entre les acteurs dans le cadre des «scènes locales » qui s'apparentent à des espaces de débats ou des «forums» (Callon, Lascoumes, Barthe, 2001), nous avons eu recours à une sociologie de type pragmatique qui combine étroitement l'observation participante, les visites de terrain, la réalisation d'entretiens et l'analyse des sources écrites. L'objectif étant de suivre au plus près, d'analyser aussi finement que possible ces processus d'action collective dans les différentes « scènes locales » (comité de pilotage, secrétariat technique, groupe de travail, etc.) où se confrontent les points de vue des différents acteurs.

L'élaboration concertée des documents d'objectifs qui correspond à une initiative française, sera tout d'abord appréhendée dans un cadre plus général, celui d'une directive européenne qui, tout en laissant une certaine latitude aux États membres (dans la délimitation des sites et les modalités de définition des mesures de gestion) impose une obligation de résultats. Dans cette perspective, nous nous attacherons à montrer dans une première partie qu'une telle procédure, fondée sur la délibération, comporte également une dimension prescriptive à travers le rôle de cadrage exercé par la puissance publique. Nous examinerons ensuite comment cette même procédure a été appropriée localement, voire en partie détournée par une fraction des acteurs hostiles à la directive, à travers la mise en place d'une démarche de coopération inter-établissements publics qualifiée d'innovante par ses protagonistes. Nous mettrons en particulier l'accent dans une troisième partie sur les choix méthodologiques adoptés. Choix qui accordent une grande autonomie à chacun et n'excluent pas, ce sera l'objet de la dernière partie, les tensions et les divergences entre coopérateurs.

\section{La procédure d'élaboration des docob : entre prescription et délibération}

Le dispositif de mise en œuvre de la DH en France peut-être schématisé en deux grandes phases : le travail de désignation des sites et l'élaboration sur chaque site d'un docob. La procédure de concertation initiée par le Ministère de l'Environnement pour établir les plans de gestion, sur laquelle porte la réflexion développée ici, vise à favoriser l'appropriation de la biodiversité à l'échelle des territoires. Elle est explicitée en ces termes : «Le docob constitue une démarche novatrice. Il est établi sous la responsabilité du préfet de département assisté d'un opérateur technique, en faisant une large place à la concertation locale. Le comité de pilotage regroupe sous l'autorité du Préfet les partenaires concernés par la gestion du site (collectivités locales, propriétaires, exploitants, associations, usagers...) ou leurs 
représentants. Ce document définit les orientations de gestion et les mesures de conservation contractuelles et indique, le cas échéant, les mesures réglementaires à mettre en cuvre sur le site. Il précise les modes de financement des mesures contractuelles » (Ministère de l'Aménagement du Territoire et de l'Environnement (MATE), 2003, p. 10) ${ }^{3}$.

Cette procédure s'inscrit à première vue dans ce qu'il est convenu d'appeler les nouvelles modalités de l'action publique marquée par la territorialisation et l'implication de nombreux acteurs. Selon Durand et Thoenig (1996), on serait passé d'un système autocentré, caractérisé par un État fort qui définissait les buts et les moyens poursuivis pour les atteindre, à un système qui s'attache à promouvoir ce qu'on pourrait appeler un "intérêt général territorialisé ». Dans ce nouveau contexte, le rôle de l'État consiste à promouvoir un éventail de procédures visant à favoriser la mise en place d'espaces de rencontres et de débats impliquant une diversité d'acteurs, à l'image des comités de pilotage évoqués plus haut. "L'institutionnalisation de l'action collective devient un processus majeur qu'adopte l'État pour peser sur la conduite des affaires publiques» (Duran, Thoenig, 1996: 600). Ces nouvelles modalités de l'action publique qualifiées également de politiques procédurales «se présentent sous la forme d'énoncés très généraux dont le sens reste à produire par la délibération collective. L'essentiel de leur contenu porte sur l'organisation de dispositifs territoriaux destinés à assurer des interactions cadrées, des modes de travail en commun et la formulation d'accords collectifs » (Lascoumes, Le Bourhis, 1998 : 39-40).

6 Cette approche en terme de politique procédurale ne saurait cependant refléter à elle seule le processus de construction des docob. Celui-ci présente en effet des caractéristiques qui relèvent à la fois du modèle des politiques « substantielles » produites par une autorité centralisée et des politiques fondées sur la délibération, à l'échelle des territoires. Certes, les docob sont co-construits au cours de débats mobilisant une pluralité d'acteurs, néanmoins le poids des pouvoirs publics demeure important dans la mise en œuvre d'une directive dont les textes prévoient une obligation de résultats. Chaque État membre est en effet tenu d'établir les dispositions nécessaires à la conservation des habitats et des espèces inventoriés sur son territoire. Dans un tel contexte, les règles qui régissent les procédures de concertation locales sont largement encadrées par l'administration centrale, les Diren et les opérateurs. La présidence des comités de pilotage par les préfets, la validation des docob par les représentants de l'État ou encore la mise au point d'outils de gestion des sites Natura 2000, constituent quelques uns des moyens mis en œuvre par la puissance publique pour assurer la réalisation des objectifs de la DH.

7 Le caractère prescriptif de la procédure de la $\mathrm{DH}$ réside également dans le fait que l'objectif affiché de préservation de la biodiversité ne relève pas d'initiatives locales mais s'est imposée peu à peu aux États par le biais de grandes négociations internationales (Pinton et al., 2005). Dans le cas français, c'est le Ministère de l'Environnement qui, par le biais de procédures locales de concertation, enjoint les acteurs du territoire à s'approprier la biodiversité. Si la conservation du vivant a acquis aujourd'hui une certaine légitimité comme en témoigne sa mise à l'agenda de nombreuses politiques publiques (DH, ORGFSH ${ }^{4}$, stratégie nationale pour la biodiversité qui prévoit, d'ici 2010, de mettre un terme à son érosion), cette préoccupation est loin d'être partagée par tous. L'intensité des controverses qui ont marqué l'étape initiale de la DH, centrée sur l'inventaire des habitats et des espèces présentes sur le territoire national et qui ont conduit en 1996 au gel de la directive par le premier ministre Alain Juppé, permet d'en rendre compte. Un bref rappel des faits nous paraît utile à ce stade pour donner à voir les évolutions qu'a connues la 
procédure au fil du temps et situer dans quel contexte le travail d'élaboration des docob a été mené.

8 L'inventaire des sites, étape préalable à la réalisation des docob, a suscité en France de vives critiques qui ont conduit à la constitution d'un fort mouvement d'opposition incarné par le "Groupe des 9 ", réunissant les principaux représentants des milieux forestiers, agricoles, cynégétiques et piscicoles. À un premier niveau d'interprétation, on pouvait y voir la manifestation d'une résistance de secteurs importants de la société rurale à une tentative de "naturalisation" des territoires sur lesquels ces acteurs déployaient leurs activités de production et de loisirs. Production et usages contre protection de l'environnement, ruralité contre urbanité. Ces classiques couples d'oppositions pouvaient sembler servir de grille d'analyse de cette crise politique sans précédent dans le domaine des politiques de conservation de la nature conduites jusqu'alors en France. Nos travaux ont cependant montré (Rémy et al., 1999 ; Alphandéry, Fortier, 2001), qu'au-delà de l'hostilité des principaux représentants de l'espace rural envers la directive, c'était la remise en cause du monopole de la science à définir de nouvelles normes de gestion des territoires qui était au centre de nombreux débats. La $\mathrm{DH}$, comme de plus en plus de politiques publiques menées dans le domaine de l'environnement, place en effet les connaissances scientifiques au cœur du dispositif de conservation, qu'il s'agisse de définir les habitats et espèces à protéger, d'identifier les sites conformes à ces objectifs, de proposer les mesures de gestion et d'évaluer les résultats de ces dernières. Or, les controverses que l'application de la DH a entraînées ont autant - sinon davantage - porté sur cette place éminente de la science - et sur son contenu - que sur les objectifs de la directive. C'est d'ailleurs à ce questionnement du monopole de la science que la procédure de concertation choisie en France, à la suite de la crise de 1996, a tenté de répondre en favorisant l'intégration d'acteurs dotés de compétences et de savoirs divers dans le travail de désignation des sites. Cette crise politique a constitué un «épisode marquant » (Dodier, 2003) dans l'histoire de la mise en œuvre de la $\mathrm{DH}$, au sens où elle a affecté plus ou moins durablement les rapports entre les différents acteurs impliqués dans la procédure, comme nous allons le voir à travers la situation observée en région Nord-Pas de Calais.

\section{La coopération en Nord-Pas de Calais, un mode de gestion des conflits}

9 Le travail d'élaboration des docob relève, selon la procédure, de structures distinctes des Diren, qualifiées d'opérateurs ${ }^{5}$. Ceux-ci recouvrent un panel diversifié d'institutions (Parcs naturels régionaux (PNR), Office national des forêts (ONF), collectivités territoriales, conservatoires des espaces naturels, associations environnementales, chambres d'agriculture, bureaux d'études, etc., $c f$. Pinton et al., 2007) qui se portent volontaires. La désignation des opérateurs a été marquée, dans la région Nord - Pas-deCalais, par d'importantes tractations qui ont conduit à l'adoption d'une procédure inédite visant à désigner non pas un mais plusieurs opérateurs pour effectuer un même docob. Cette innovation procédurale s'inscrit dans un processus très conflictuel, qui ne peut être compris indépendamment des évènements survenus au cours de la phase d'inventaire, en particulier les tensions exacerbées entre les représentants de la forêt privée et les scientifiques chargés de désigner les périmètres Natura 2000. 
10 Comme à l'échelon national, les représentants de la forêt privée ont été les premiers à se mobiliser à propos de la DH dans la région. Dès l'automne 1994, ils ont multiplié les démarches pour prendre connaissance des inventaires. Le récit d'un responsable de la forêt privée Nord Picardie permet de comprendre la généalogie de la contestation au niveau local. "Dans le NPC, j'avais récupéré les inventaires en juillet 1995 et là, on n'a pas été déçu. On s'est aperçu que $85 \%$ des surfaces forestières privées étaient dedans (...). En Picardie comme dans le NPC on a fait des contre-propositions de sites. On les a données au préfet de région avant l'envoi à Paris. La proposition que l'on avait faite dans le NPC est effectivement partie à Paris, ça, j'en suis sûr. Par contre elle a été mise à la poubelle à Paris (...) Y’a eu la fameuse réunion $d u$ [Conseil National de la Protection de la Nature] CNPN en mars 1996 qui a examiné l'ensemble des sites. Concernant le NPC (...) ils se sont prononcés sur l'inventaire des scientifiques et notre proposition avait disparue ». La non prise en compte des choix formulés par les forestiers marque le début d'une période d'affrontements entre les représentants de la forêt privée et les scientifiques du Conservatoire botanique de Bailleul en charge de la délimitation des périmètres, l'une des principales sources de ce conflit étant la remise en cause du droit de propriété. Les scientifiques ont outrepassé les règles en vigueur en pénétrant sur les terrains privés sans autorisation, contrairement à ce que prévoit la loi ${ }^{6}$.

11 En outre, en se déclarant les portes-paroles d'un bien commun (la biodiversité), les scientifiques ont mis l'accent sur la nécessaire redéfinition des droits et des devoirs de chacun vis-à-vis de la propriété privée. Les propositions de la Ministre de l'Environnement de redéfinir les périmètres en accord avec l'ensemble des partenaires concernés n'ont pas mis fin à ces divergences. Le clivage entre forestiers privés et scientifiques, loin de s'estomper a, au contraire, gagné en puissance, en se déplaçant sur le terrain des compétences. Pour faire face aux accusations portées par les forestiers sur le manque de rigueur des naturalistes dans le premier travail de réalisation des inventaires ${ }^{7}$, ces derniers se sont repliés sur la science et ont fait montre d'une grande finesse du point de vue de l'analyse au point de rendre le dialogue impossible avec les représentants de la forêt privée. Un membre du Conservatoire de Bailleul analyse le conflit en ces termes : "Ce qui a été mal perçu, c'est que d'autres personnes, d'un autre monde si on peut dire, viennent leur donner des leçons de gestion (...). Quelque part, ils voulaient nous remettre en cause, montrer qu'ils connaissaient alors qu'en fait ils ne connaissaient pas. X qui était beaucoup plus revendicatif que moi leur a montré clairement qu'ils n'étaient pas compétents dans le domaine; c'était nous qui détenions les compétences. Mr Y a dit l'autre fois à mon collègue qui est allé faire des prospections dans ces forêts que nous étions des ayatollahs scientifiques (rire). C'est complètement renverser les choses. Simplement on a une cohérence. Je crois que c'est une rigueur et une cohérence par rapport à une démarche qui est toujours la même. On ne déroge pas. C'est ce qui est considéré comme étant jusqu'auboutiste». Les forestiers, de leur côté, ne se sont pas privés de mettre l'accent sur le caractère incertain et non stabilisé des connaissances liées à la préservation de la biodiversité et sur les divergences de point de vue au sein de la communauté scientifique (Alphandéry, Fortier, 2005). Enfin, l'intensité des conflits dans la région tient également aux responsabilités détenues par quelques grands propriétaires locaux dans les instances sylvicoles nationales et régionales. L'un des principaux pourfendeurs de la DH au niveau national appartient, en effet, à une famille de propriétaires bien connue du Nord de l'hexagone.

12 Cantonné dans un premier temps aux rapports entre scientifiques et forestiers privés, le conflit s'est étendu à la faveur des événements nationaux (publication des listes par le CNPN, instauration du "groupe des neuf » en avril 1996) à d'autres catégories d'acteurs 
de l'espace rural en particulier les chasseurs, les agriculteurs, les propriétaires de dunes. La création, à l'initiative des forestiers privés, de deux associations hostiles à la $\mathrm{DH}$, Natura 200062 dans le Pas de Calais et Natura 200059 dans le Nord, soit l'équivalent du " groupe des neuf » à l'échelon départemental, marque l'intensité de la contestation dans le Nord-Pas de Calais (Fortier in Rémy et al., 1999). Ces associations, qui n'ont pas d'équivalent dans les autres régions, ont exercé un pouvoir de lobbying très important auprès de la préfecture au point de s'imposer comme les acteurs clefs du processus de renégociation des périmètres proposé par la Ministre de l'Environnement en réaction au "groupe des neuf ». Comme l'a fait très justement remarquer un gestionnaire d'espace protégé, " on a assisté à un renversement complet de tendance ", à une inversion des rapports de force. Les scientifiques, tout au moins dans un premier temps, ont été évincés à leur tour de la négociation au profit des représentants des associations Natura 2000. La conséquence en fut une réduction considérable de l'étendue des superficies proposées au titre de la DH: de 13,4\% en 1996, elle représente 1,47\% du territoire [Domaine Public Maritime (DPM) inclus], en $2005^{8}$.

Le lancement des premiers docob a débuté tardivement dans la région. C'est seulement au cours de l'année 2000, soit près de deux ans après le lancement de la procédure par la Ministre de l'Environnement d'alors, qu'ont été signées les premières conventions entre les services de l'État et les co-opérateurs. Le choix initial de la Diren de recourir à un appel d'offres pour désigner les opérateurs a été vivement rejeté : par les PNR tout d'abord, qui s'estimaient légitimes pour être opérateurs sur «leur» territoire", par les associations Natura 2000 ensuite, qui voyaient d'un mauvais œil le recours possible à des bureaux d'études environnementalistes, extérieurs au territoire ou encore la désignation d'associations à caractère naturaliste comme le Conservatoire botanique de Bailleul, le Conservatoire des sites naturels et la Fédération Nord-nature. Il est vrai que la proposition de mise en concurrence de différentes structures par la Diren, peu utilisée dans les autres régions (Pinton et al., 2007), et présentée comme une nécessité du point de vue des règles du marché public, répondait aussi à une volonté de la part de l'administration régionale de l'environnement de redonner une place aux scientifiques et aux naturalistes contraints de céder aux pressions importantes des associations Natura 2000. En dépit de la faiblesse des superficies concernées par Natura 2000 dans le Nord-Pas de Calais, le statut d'opérateur s'est avéré être un enjeu pour les acteurs socioprofessionnels (Chambre d'agriculture, Centre régional de la propriété forestière (CRPF), par ailleurs membres des associations Natura 2000). Il s'agissait de contrecarrer le projet de la DIREN et dans le même temps de se faire reconnaître comme opérateurs légitimes. "La protection de la nature ce n'est pas que l'affaire des écolos et des scientifiques souligne un responsable de la Fédération départementale des syndicats d'exploitants agricoles (FDSEA). Nous, on en vit de la nature. Enfin, on occupe le terrain et on veut montrer qu'on est capable de faire des choses. (...) On a besoin d'afficher qu'on s'investit dans ce travail. Et pas seulement en terme d'affichage, pour montrer qu'on a vraiment une compétence et qu'on est capable de faire des choses "

14 Une telle décision a pu apparaître comme un changement de positionnement de la part des associations Natura 2000 par rapport à la période des inventaires. Ceux qui s'étaient montrés hostiles à la DH manifestaient dès lors le désir de s'impliquer, d'être acteur dans la procédure. Toutefois, ces deux attitudes ne sont pas contradictoires. Les opposants à Natura 2000 ont fait le choix de s'investir dans la procédure pour mieux la contrôler ${ }^{10}$. À l'issue de nombreuses tractations menées en coulisse avec les Parcs et les services de 
l'État, les associations Natura 2000 ont obtenu des pouvoirs publics (de la préfecture en particulier auprès de laquelle ils disposent d'un fort pouvoir d'influence) que la réalisation des docob soit confiée exclusivement à des structures publiques ${ }^{11}$, avec la possibilité de désigner plusieurs opérateurs pour réaliser un seul et même docob. La mise en place de cette procédure inédite était loin de faire l'unanimité. Elle reflétait avant tout la volonté du CRPF et de la Chambre d'Agriculture d'exercer un contrôle sur le travail réalisé étant donné le manque de confiance vis à vis des $\operatorname{Parcs}^{12}$ ou d'autres structures jugées trop proches des associations de protection de la nature. "On veut tenir le crayon " soulignait avec insistance un responsable de la forêt privée. «En tenant la plume (...) on peut faire en sorte qu'il y ait un équilibre entre les enjeux socio-économiques et les enjeux de conservation de la biodiversité». La position des Parcs se voulait quant à elle plus nuancée tout en réaffirmant leurs compétences pour être maîtres d'œuvre des docob: "Nous, on n'a jamais voulu être opérateur seul sur les sites. On considère que ce n'est pas notre manière de travailler. Vu le contexte politique qu'il y avait à l'époque et qui existe toujours, cétait aller au casse-pipe (...) d'autant que nous étions en révision de charte et que le président du PNR disait clairement aux élus : le Parc c'est le meilleur paravent contre Natura 2000 car il va favoriser la concertation». Et ce membre des services techniques du Parc d'ajouter: "De plus, on ne voulait pas laisser la Chambre [d'agriculture] et le CRPF partir seuls sur la rédaction des Docob. On considère que sur la gestion des milieux naturels et la concertation on a davantage de compétences qu'eux ».

15 Si la démarche de coopération était volontiers qualifiée d'innovante par ses principaux instigateurs (CRPF, Chambre d'agriculture) et disposait a priori d'un certain nombre d'atouts (élargissement des compétences, reconnaissance de la légitimité de chacune des structures impliquées, facilitation du travail de terrain et des actions de communication), elle devait être avant tout envisagée, dans le cas précis, comme un instrument de gestion des conflits institutionnels. Dans un courrier adressé au Préfet du département du Nord, le chargé de mission de la DIREN faisait ainsi état des «enjeux de pouvoirs susceptibles de rendre particulièrement difficile la réalisation des docob $»^{13}$. La décision de confier le travail d'élaboration des docob aux principaux représentants des gestionnaires locaux ne résultait donc pas tant de la volonté des partenaires de collaborer à une meilleure prise en compte de la nature dans les activités humaines, qu'à un souci de limiter les rivalités et les phénomènes de concurrence entre les structures opératrices. Entre le projet initial de la DIREN d'organiser une procédure d'appel d'offres et la décision finale de restreindre les opérateurs aux seuls établissements publics, on a assisté à un véritable coup de force de la part des associations Natura 2000 destiné à exclure les structures naturalistes (associations de protection de la nature, bureaux d'études) du statut d'opérateur. Les établissements publics ont eu dès lors un statut de monopole tout au moins sur les sites localisés en zone de Parcs ${ }^{14}$.

\section{Principes de mise en œuvre du docob en région Nord-Pas de Calais : cloisonnement institutionnel et partition territoriale}

16 L'élaboration conjointe d'un même docob a donné lieu, là encore, à de nombreux débats sur la méthode à utiliser. Comment procéder? Quels principes de fonctionnement fallaitil adopter? Après de multiples discussions largement orchestrées par la Chambre d'agriculture et le CRPF il a été acté que chacun aurait en charge la portion de territoire 
dont il est le gestionnaire ou le représentant. Cette partition de l'espace fondée sur des critères de propriété et de type d'activité a conduit dans le cas précis du site NPC 11 (Pelouses et forêts calcicoles de la Cuesta-sud du Boulonnais) ${ }^{15}$ à confier au CRPF l'ensemble de la propriété forestière privée, à la Chambre d'agriculture les terrains agricoles, les territoires des collectivités publiques revenant aux deux structures impliquées dans la gestion des milieux naturels: Eden $62^{16}$ et le PNR ${ }^{17}$. Ce parti-pris méthodologique s'est doublé d'une autre exigence: "Chacun (...) rédige les éléments $d u$ document qui le concerne et anime les réunions et visites nécessaires à la rédaction des parties qui lui incombe ${ }^{18}$. En d'autres termes, chaque opérateur, à quelques nuances près, s'est vu confier pour l'espace dont il a la charge, un ensemble de tâches établies en référence à un cahier des charges élaboré par les soins de la DIREN (réalisation d'études socioéconomiques, inventaire et cartographie des habitats, définition et hiérarchisation des enjeux, élaboration des mesures de gestion) avec la possibilité de réaliser lui-même ces travaux ou de faire appel à des prestataires extérieurs. La responsabilité accordée à chaque opérateur de maîtriser l'ensemble du processus sur "son » territoire n'excluait pas cependant un droit de regard sur le travail d'autrui. L'ensemble des documents produits et des synthèses effectuées sur chaque portion d'espace (regroupée en deux types de milieux : la forêt et les milieux ouverts) était soumis à discussion dans le cadre d'une instance baptisée "secrétariat technique ». Ce collectif qui réunissait les équipes techniques de chacune des structures opératrices ${ }^{19}$ avait pour mission de « rassembler et d'harmoniser les éléments apportés par les membres et leurs sous-traitants, afin d'assurer la transversalité et le liant nécessaire à des documents d'objectifs de qualité ${ }^{20}$ et, au-delà, de répartir les tâches entre opérateurs, définir la stratégie de communication, la nature des éléments à rédiger, etc. L'animation du secrétariat technique était confiée au CRPF.

Ces principes de fonctionnement fondés sur une division sectorielle du travail, loin de favoriser l'intégration des différents points de vue, intérêts, valeurs et rationalités, ont à l'inverse conforté les uns et les autres dans leur propre logique, avec la crainte parfois de se voir imposer un certain nombre de prérogatives. Un technicien d'Eden 62 dont la mission était de concilier la préservation des milieux naturels et l'accueil du public sur les terrains du Conseil Général ne cachait pas ses inquiétudes et la difficulté de cette entreprise. "C'est un changement de méthode de travail parce qu'on est obligé de confronter nos opinions et notre façon de faire à l'avis des autres. C'est très dur ». D'autant que les objectifs et la stratégie adoptés par les uns et les autres étaient loin d'être identiques. « Nous, poursuit ce même technicien, on défend la politique des espaces naturels sensibles; il est hors de question que sur ces espaces-là sur lesquels on intervient depuis longtemps, sous couvert de Natura 2000, on fasse autre chose qui serait proposé par le CRPF, la Chambre [d'agriculture] et qui pourrait transgresser les objectifs que l'on vise depuis plusieurs années (...). On a quand même un peu d'amour propre et l'espoir de ne pas s'être trompé dans les objectifs. Sachant que c'est sur la base d'inventaires scientifiques que l'on travaille». L'implication des acteurs socioprofessionnels dans ce processus était, selon lui, très différente : « Pour le CRPF, la Chambre [d'agriculture], ils participent aux docob, ils affichent une volonté d'appliquer Natura 2000 mais avec l'arrièrepensée qu'ils ont quand même à faire référence aux autres acteurs de Natura 200062 et l'arrièrepensée de minimiser l'impact de Natura 2000 sur leurs terrains ». Quant au PNR il occupait une position plus délicate. «Il a le cul entre deux chaises, faisait remarquer notre interlocuteur. Premièrement, il doit montrer un minimum d'écoute, de concertation avec les autres acteurs: Eden, la Chambre[d'agriculture], le CRPF et, deuxièmement, comme il est établissement public qui affiche 
ses politiques et ses résultats, il doit faire attention de ne pas trop montrer de souplesse par rapport à ces acteurs aux dépens des associations de protection de la nature ».

En définitive, on a assisté au fil de l'avancement du docob à une sorte de modus vivendi entre les opérateurs qui les a conduit à se montrer relativement indulgents les uns vis-àvis des autres, évitant ainsi de voir leurs propres pratiques remises en cause. Aucun d'eux n'avait intérêt à agiter le "chiffon rouge " au risque de provoquer une situation de blocage. "On ira jusqu'à la rédaction des docob sinon on est obligé de rembourser l'État" soulignait l'un d'eux. À première vue, cette expérience de coopération n'a pas induit de changements significatifs dans les conduites des acteurs. Le principe du « chacun chez soi » adopté dès le départ de la procédure n'a pas été remis en cause. Il a prévalu tout au long du travail d'élaboration du document d'objectifs et ce, en dépit de l'existence de thèmes transversaux communs mais qui n'ont pas été abordés comme tels, à l'exemple de la question des lisières ou encore de l'activité cynégétique qui représentait l'un des principaux enjeux socio-économiques dans ces terrains pentus, difficilement exploitables. Les opérateurs se sont à des degrés divers cantonnés dans des positions défensives avec bien sûr des périodes relativement apaisées et d'autres caractérisées par des temps forts, des blocages, des débordements. Mais pour autant ne s'est-il rien passé ? Et que révèle plus fondamentalement l'expérience du Nord-Pas de Calais du point de vue de la mise en œuvre des procédures de concertation? C'est ce que nous allons voir à présent à travers l'examen de quelques controverses.

\section{Quelques points de désaccord}

Le travail d'élaboration du document d'objectif sur le site NPC11 a révélé un certain nombre de points de désaccord entre les opérateurs. Nous avons choisi d'en exposer trois. Le premier concerne le jeu des acteurs et soulève de façon plus générale la manière dont des institutions telles que la Chambre d'agriculture et le CRPF se positionnent à l'égard de ces nouvelles dispositions en faveur de la biodiversité. Le second pose la question de la légitimité de certaines structures à investir des missions qui ne leur sont pas normalement dévolues. Enfin, nous terminerons en évoquant les tensions suscitées à propos des conditions de mise en œuvre de la procédure et tout particulièrement le manque de moyens financiers alloués pour mener à bien cette politique.

\section{La « double casquette » de la Chambre d'agriculture et du CRPF}

La coopération inter-établissements publics a conduit, on l'a vu, à évincer un certain nombre de structures du statut d'opérateur, dont l'association Natura 200062 . Néanmoins, celle-ci n'a pas désarmé. Elle a tenté d'obtenir une implication effective dans le processus d'élaboration du docob en proposant la signature d'une convention avec l'État et les autres opérateurs. Proposition qui a été vivement rejetée à plusieurs reprises par Eden 62 au motif que Natura 200062 s'était montrée particulièrement hostile à la directive en réduisant de façon drastique les périmètres lors de la phase d'inventaire. La position du Parc était elle aussi emprunte d'une certaine réserve. "On ne voit pas pourquoi l'association devrait figurer dans cette convention, ce sont avec les établissements publics que l'on traite pour établir les docob et pas avec Natura $200062 \%$. Et l'un de ses techniciens d'ajouter : "L'association cherche à s'intégrer dans une procédure qui est cadrée et pour laquelle on a une obligation de résultats ». Cette décision n'a pas empêché la Chambre d'agriculture et le CRPF 
d'intervenir à double titre au sein des instances de débat: tantôt en qualité d'établissements publics, tantôt en tant que membres représentant les intérêts de l'association. D'où les tensions au sein du secrétariat technique qui se sont cristallisées lors de la rédaction du premier bulletin d'information. De profonds désaccords sont apparus du point de vue des messages à faire passer dans ces documents. "À propos de l'édito, faisait remarquer un membre d'Eden, au lieu de relater le travail de coopération, ils voulaient mettre en avant l'opinion des structures, autrement dit émettre toute une série de réserves sur Natura 2000 ». Constat similaire de la part d'un technicien du Parc: «Dans la première lettre d'information, ils [le CRPF et la Chambre d'agriculture] voulaient mettre en avant la composition de Natura 200062 ! Là aussi ça a donné lieu à débat. Nous, Parc, on était d'accord mais pas Eden. Qu'ils fassent figurer une interview du président de l'association à un moment donné, d'accord, mais qu'il y ait dans le premier numéro, une volonté d'imposer presque aux forceps la présence de l'association, ça montre bien la volonté de mainmise sur le truc ».

21 L'attitude ambivalente de la Chambre d'agriculture et du CRPF de jouer tour à tour sur l'un et l'autre registre manifestait une volonté de participer à la démarche, étant donné l'importance croissante accordée à la biodiversité dans les politiques publiques, tout en s'efforçant de ménager leurs mandants qui étaient pour une fraction d'entre eux hostiles à la DH. Cette forme d'engagement qui mêlait étroitement implication et évitement révélait plus fondamentalement les clivages internes à la profession agricole et forestière suscités par l'adoption de ce nouveau référentiel. La gestion de la biodiversité, notamment dans les espaces de "nature ordinaire", implique en effet de promouvoir d'autres formes de rationalité, de repenser des modèles de développement compatibles avec la préservation des habitats et des espèces. Ces changements qui contribuent à redéfinir les métiers d'agriculteur ou de sylviculteur sont à l'origine de débats parfois très vifs, souvent difficiles à gérer (Fleury et al., 2003).

\section{La légitimité contestée du CRPF dans l'acquisition de compétences naturalistes}

La volonté du CRPF d'acquérir des compétences naturalistes a été à l'origine de nombreuses tensions entre forestiers et naturalistes. Ces divergences interrogent la légitimité des acteurs de la forêt privée à disposer d'une capacité d'expertise dans ce domaine et renvoient plus fondamentalement à la question du monopole de l'expertise. L'implication du CRPF dans l'élaboration du docob a constitué une opportunité pour ce dernier de développer des connaissances à l'interface entre la forêt et l'environnement. L'institution se définissait alors selon les termes de son directeur comme un « assembleur de compétences ». Outre sa légitimité d'établissement public dans le domaine de la forêt privée, le CRPF ambitionnait d'acquérir des connaissances en phytosociologie, botanique forestière, pédologie forestière de manière à articuler la production et l'économie forestière avec les nouveaux enjeux de conservation de la biodiversité. Cette volonté était ainsi justifiée par l'un de ses membres : "S'il n'y a que quelques personnes en France qui sont phytosociologues émérites et qui sont seuls capables de juger de la qualité des milieux, on n'aboutit pas à l'objectif. Il faut que chaque opérateur réussisse à s'approprier ces notions-là (...) ça ne doit pas rester un domaine d'experts ». Mais l'acquisition de compétences écologiques en interne pouvait être aussi appréhendée comme une volonté de limiter l'intrusion des scientifiques naturalistes sur les terrains privés. Après les inventaires des Zones naturelles d'intérêt écologiques floristiques et faunistiques (ZNIEFF) puis la délimitation 
des périmètres Natura 2000, à l'origine de vives polémiques, les représentants de la propriété privée de la région ont mis un point d'honneur à faire en sorte que les travaux d'inventaires complémentaires, effectués dans le cadre des docob, soient réalisés par leur soin, en toute légalité, avec autorisation préalable adressée à chaque propriétaire de pénétrer dans sa ou ses parcelles. Le développement des connaissances naturalistes de la part des forestiers constituait dès lors le meilleur moyen de défense de la propriété privée, comme le suggérait un gestionnaire d'espaces naturels: "Le CRPF et de façon générale Natura 200062 s'attribuent la propriété des plantes qui sont sur leurs parcelles et donc l'info qui relate la présence de telle ou telle espèce ou habitat leur appartient. Et donc ils sont les seuls à pouvoir décider de diffuser ou pas cette information, alors qu'en réalité il s'agit d'un patrimoine naturel, d'un bien commun ».

La décision prise par le CRPF de ne pas faire appel aux experts scientifiques locaux, mais de privilégier le recrutement $d$ ' "un stagiaire encadré par un scientifique de renom » pour réaliser les inventaires et la cartographie des habitats forestiers, a suscité des réactions très mitigées voire indignées de certains acteurs impliqués dans la conservation de la nature, au motif que ce stagiaire n'avait pas les compétences requises ${ }^{21}$. À diverses reprises, l'opérateur s'est vu interpellé dans les instances de débats (secrétariat technique, comité de pilotage) afin d'apporter des informations sur le protocole adopté ou préciser tel ou tel aspect relatif à l'identification des habitats et leur cartographie. Mais c'est au cours de la phase finale, lors de l'évaluation du docob par le conseil scientifique régional du patrimoine naturel (CSRPN), que les critiques adressées ont été les plus virulentes. Le rapport a en effet mis l'accent sur de nombreuses insuffisances: absence de finesse de l'analyse, non prise en compte de certains milieux, notamment les milieux associés aux habitats forestiers, manque de corrélation entre les pratiques de gestion du milieu, la nature et l'état de conservation des habitats. Les représentants du CSRPN ont également dénoncé le recours trop systématique aux mesures préconisées dans les Cahiers d'Habitats au détriment de la prise en compte des spécificités locales. L'homogénéisation des pratiques allait, selon eux, à l'encontre des objectifs visés par la directive, à savoir le maintien voire le développement de la biodiversité. Ce travail d'évaluation, qui s'est apparenté à une sorte de compte rendu d'experts à l'encontre de praticiens «débutants » ou assimilés, à été l'occasion de réaffirmer à l'ensemble des opérateurs et au CRPF en particulier "la complexité de la phytosociologie, discipline nécessitant de nombreuses années d'expérience pour en maîtriser tous les concepts et les applications concrètes $»^{22}$. Après avoir été fortement décriés par les acteurs socioprofessionnels à propos du manque de fiabilité de leur travail lors de la phase d'inventaire, les scientifiques ont tenu à ce stade, à réaffirmer leurs savoirs et leurs compétences dans un domaine qu'ils considèrent comme n'étant pas à la portée de «tout le monde ».

\section{Le déficit de moyens, un frein à l'engagement des forestiers}

Enfin, la question des conditions de la mise en œuvre de la procédure, en particulier le manque de moyens alloué pour financer les mesures de gestion a, elle aussi, alimenté de vives discussions au sein du secrétariat technique. La polémique est apparue au lendemain de la diffusion de la circulaire du Ministère de l'Environnement, au printemps 2003, qui dressait un état des lieux des sources de financement largement revues à la baisse, notamment dans le domaine forestier. Le manque de crédits disponibles ne 
permettait pas de mettre en place des outils nouveaux, adaptés à ce secteur. Cette circulaire a déclenché une profonde amertume chez les représentants de la forêt privée qui ont dénoncé une fois encore l'incapacité de l'État à tenir ses engagements. «Comment on rédige un document dès lors qu'on ne sait même pas si y'aura des crédits! Comment peut-on afficher quelque chose d'alléchant, d'incitatif alors qu'on ne sait même pas qui finance et à quel taux! Moi, en forêt, je ne sais pas (...). Je me mets à la place du propriétaire... si demain, on peut financer la création d'une marre uniquement à $50 \%$, le propriétaire va-t-il signer un contrat?" (Un technicien du CRPF). Se sentant désavoués, les représentants de la forêt privée ont alors décidé de ne faire figurer dans le docob que les mesures finançables ou dépourvues de contraintes. Cette décision a été vivement désapprouvée par les gestionnaires d'espaces naturels (le Parc et Eden) qui la jugeaient incompatible avec l'objectif même des docob : garantir la sauvegarde des habitats et des espèces présents dans les sites Natura 2000. La question n'ayant pu être résolue au sein du secrétariat technique, elle a finalement été arbitrée par la Diren et tranchée en Comité de pilotage. Tout en reconnaissant les difficultés de la situation, les services de l'État, appuyés par le représentant du CSRPN, ont tenté de convaincre le CRPF de raisonner par objectif sans préjuger de ce qui serait ou non finançable. «La priorité souligne la DIREN, c'est d'abord de définir les actions et ensuite on verra quels financements on peut trouver ». À la différence des situations précédentes c'est l'État cette fois qui est montré du doigt et accusé de ne pas fournir les conditions indispensables (moyens financiers, stabilisation des conditions de mise en œuvre de la procédure) à l'engagement des acteurs dans le processus et à l'appropriation par ces derniers de la biodiversité.

\section{Conclusion}

L'expérience de coopération (association d'opérateurs en vue d'élaborer un docob) développée en région Nord-Pas de Calais ne vise pas tant la création d'une dynamique institutionnelle autour des objets de nature comme on serait tenté de le penser, mais apparait comme une réponse négociée entre quelques institutions pour tenter d'apaiser les conflits et mettre un terme au blocage de la procédure. Cette forme de partenariat "par défaut» s'inscrit en outre dans une tentative d'éviction des scientifiques naturalistes de l'élaboration des docob et révèle plus largement les clivages existants entre ces derniers et certains acteurs socioprofessionnels: propriétaires forestiers, agriculteurs, chasseurs, regroupés au sein d'associations départementales (Natura 200059 et 62), hostiles à la DH. Ce clivage transparaît à toutes les étapes du processus de mise en œuvre de la directive, depuis la phase d'inventaires jusqu'au stade de validation du docob où les scientifiques, écartés du débat, opèrent un véritable retour en force en dénonçant les insuffisances du travail réalisé par les coopérateurs. Ainsi, par une sorte d'effet de balancier, on assiste à un conflit sans fin où chacune des parties se sent évincée et tente d'évincer l'autre en retour. Cette situation paraît d'autant plus paradoxale dans une région où la superficie consacrée au futur réseau Natura 2000, au titre de la DH, n'excède pas $1,47 \%$ du territoire (DPM inclus). Elle trouve cependant sa raison d'être dans les conflits exacerbés qui se sont déroulés au cours de la définition des périmètres entre les scientifiques et les représentants de la forêt privée à propos de la question très délicate des droits attachés à ce type de propriété. Dans ces conditions, le partenariat engagé, loin de favoriser le dialogue, a contribué à attiser les tensions et les rapports de force. Chacun des acteurs impliqués s'est cantonné dans un jeu de rôle qui a fait obstacle à un véritable 
travail de collaboration. Les acteurs socioprofessionnels (représentants de la forêt privée et de l'agriculture) ont justifié leur participation au nom de la défense des intérêts de leurs mandants, l'administration s'est dite dans l'incapacité d'agir étant donné le poids politique des associations Natura 2000 auprès de l'autorité préfectorale. Quant aux scientifiques, ils se sont repliés sur la science en faisant montre d'une grande exigence à propos du travail accompli par les coopérateurs, réaffirmant ainsi leur savoir-faire et leur légitimité fortement mis en cause au stade de l'inventaire.

Si le docob a, en définitive, été validé par l'autorité préfectorale, le processus d'élaboration conjointe du plan de gestion n'a pas permis l'instauration d'un véritable dialogue entre les différentes structures engagées. L'hypothèse que nous formulions au départ, dans le cadre de l'équipe Grenat, d'une reconfiguration des rapports entre les différents acteurs, à l'occasion de l'élaboration des docob, n'a donc pas eu lieu dans le Nord-Pas de Calais. Certes, la démarche de coopération mise en avant dans la région a favorisé certains types de rapprochements, notamment entre forestiers et agriculteurs, mais elle a dans le même temps contribué à renforcer durablement les clivages entre les principaux gestionnaires de l'espace rural (forestiers, agriculteurs, chasseurs, par ailleurs membres des associations Natura 2000) et les scientifiques. La réactivation de ces associations à l'occasion de la mise en œuvre d'une disposition en faveur de la conservation de la faune sauvage et de ses habitats (ORGFSH) en 2005, souligne la persistance dans le temps de profondes divergences entre les deux groupes et les liens ténus créés entre certains représentants de l'espace rural dans le cadre de Natura 2000. Si cette expérience de travail en commun n'a pas permis aux différentes parties en présence de nouer des alliances et de parvenir à construire des compromis, elle a néanmoins induit, notamment chez les forestiers, une volonté d'acquérir et de développer des connaissances nouvelles en interne, en lien avec ce nouveau référentiel de la biodiversité. Ce phénomène nous paraît intéressant à noter parce qu'il va à l'encontre du processus d'écologisation ${ }^{23}$ qui, selon certains auteurs (Mougenot, Roussel), consiste précisément à établir des chaînes de coopération entre les différentes catégories d'acteurs et les objets de nature. La démarche de coopération inter-établissements publics affichée dans la région répond davantage à une volonté de contrôle et de maîtrise de la procédure qu'au souhait de travailler en commun. La preuve, en optant pour une division du travail par secteur d'activité, les opérateurs ont plutôt misé sur le repli sur soi et la stratégie de l'évitement que sur l'ouverture et la confrontation aux autres.

Plus généralement, cet exemple révèle, au-delà des idées reçues, que la mise en place de dispositifs délibératifs ne suffit pas à rendre possible des accords entre les différents protagonistes. L'élaboration de compromis suppose que soient réunies un certain nombre de conditions, à commencer par l'existence d'un minimum de confiance entre les différents acteurs. L'épisode emblématique des inventaires, effectué sans concertation, a ébranlé durablement la confiance entre les acteurs. Les conflits qui en ont résulté et qui sont pour l'essentiel des conflits de légitimité, se sont avérés difficiles à dépasser. Le rapport de force s'est progressivement imposé au détriment du dialogue et de la concertation. La sur-représentation des acteurs socioprofessionnels, en particulier des forestiers privés et des agriculteurs dans les comités de pilotage ou encore la non prise en compte des savoirs ordinaires portés par les acteurs de terrain sont autant de manifestations de ce rapport de force. En d'autres termes, les procédures d'actions concertées peuvent s'avérer, comme dans le cas présent, peu propices au débat, dès lors qu'elles font l'objet d'une appropriation de la part de certains groupes de pression. 


\section{BIBLIOGRAPHIE}

Alphandéry P., Fortier A., 2005, « Négociation autour de la biodiversité : la mise en œuvre de Natura 2000 en France », in Marty et al., (eds), Biodiversité : quelles interactions entre sciences de la vie et sciences de l'homme et de la société?, CNRS Ed., p. 227-240.

Alphandéry P., Fortier A., 2001, "Can a territorial policy be based on science alone ? The system for creation the Natura 2000 Network in France", Sociologia Ruralis, 41, 3, 311-328.

Barbault R., Chevassus B., (dir.), 2005, Biodiversité et changements globaux. Enjeux de société et défis pour la recherche, éd. ADPF/Ministère des Affaires étrangères, $244 \mathrm{p}$.

Blatrix, C., 2002, « Devoir débattre », in Politix, Revue des Sciences Sociales du Politique 57, p. 79-102.

Blondiaux L., Sintomer Y., 2002, « Démocratie et délibération », in Politix, Revue des Sciences Sociales du Politique 57, 17-35.

Callon M., Lascoumes P., Barthe Y., 2001, Agir dans un monde incertain, Seuil, Paris.

Duran P., Thoenig J.C., 1996, «L'État et la gestion publique territoriale », Revue Française des Sciences Politiques, 46, 4, 580-623.

Dodier N., 2003, Les leçons politiques de l'épidémie de Sida, Paris, EHESS.

Fleury P., Larrère R., Dorioz J-M., Guiseppelli E., Jullian C., Birck C., 2003, « Gestion concertée de la biodiversité, agriculture et développement local en montagne », Montagnes Méditerranéennes, 18, 65-74.

Lascoumes P., Le Bourhis J.P., 1998, « Le bien commun comme construit territorial », Politix, 42, 37-66.

Mermet L., 2004, « Ouvrir de nouveaux espaces critiques : clarifier, renouveler, « pluraliser », les ancrages normatifs des recherches », http://www.rgte.centrecired.fr/rgte/article.php3? id_article $=77$.

Mougento C., Roussel L., 2005, "To poison or to trap ? The ecologisation of "pest" control", Sociologia Ruralis, 45, 115-129.

Natura 2000, Dix questions, dix réponses, MATE, janvier 2003

Talvin J., 2003, «Les inventaires scientifiques ne sont pas toujours légaux », Forêts de France, 468, p. $40-42$.

Pinton F. (Resp. Scient.), Alphandéry P., Billaud J.-P., Deverre C., Fortier, A., Gesniaux G. (avec la collaboration de C. Lefebvre) 2007, La construction du réseau Natura 2000 en France, La Documentation française, $254 \mathrm{p}$.

Rémy E. (coord.), Alphandéry P., Billaud J-P., Bockel N., Deverre C., Fortier A., Kalaora B., Perrot N., Pinton F., 1999, La mise en directive de la nature. De la directive Habitats aux prémices du réseau Natura 2000, Rapport pour le MATE, 273 p. 


\section{NOTES}

1. Cette recherche est le fruit d'un travail collectif entrepris par le groupe de recherches sur Natura 2000 (Grenat), (Pinton et al., 2005).

2. Sur les différentes appellations attribuées à ces procédures (concertation, délibération, consultation, participation) et le sens accordé à ces notions voir en particulier Callon, Lascoumes, Barthes, 2001 et Blondiaux, Sintomer, 2002.

3. La loi sur le Développement des Territoires Ruraux (DTR) adoptée en 2005 a introduit un certain nombre d'innovations dans la procédure. Elle renforce notamment le rôle des collectivités locales dans le processus d'élaboration des docob.

4. Orientations régionales de gestion de la faune sauvage et des habitats adoptées dans le cadre de la loi chasse de 2000.

5. Les opérateurs (avec le soutien de chargés de mission) ont notamment la responsabilité de constituer et d'animer des collectifs de travail, de réunir et de mettre en forme les connaissances relatives aux savoirs locaux et à la gestion des habitats et des espèces et donc de permettre l'articulation entre les données scientifiques et naturalistes et les projets territoriaux développés par les acteurs.

6. Un inventaire ne peut être réalisé sans l'accord du propriétaire ou la signature d'un arrêté préfectoral autorisant les scientifiques à pénétrer sur la propriété. Pour connaître les textes de loi qui régissent la réalisation des inventaires, se reporter à J. Talvin, Forêts de France, $\mathrm{n}^{\circ} 468$, novembre 2003.

7. Il leur est notamment reproché de ne pas avoir procédé à un travail de terrain mais d'avoir réalisé les inventaires à partir de données bibliographiques lacunaires et non actualisées.

8. Les sites désignés au titre de la DH couplés avec les sites de la directive Oiseaux constituent le réseau écologique Natura 2000.

9. Présents au nombre de trois, les PNR disposent d'un poids politique important dans la région.

10. Cette situation rejoint le constat effectué par C. Blatrix (2002) à propos de la mobilisation associative dans des contextes participationnistes.

11. Cette décision a cependant eu pour effet d'écarter certaines composantes des associations Natura 2000 du statut d'opérateur, comme les fédérations de chasse qui relèvent du statut associatif.

12. Et ce, en dépit de l'expérience de partenariat entre la Chambre d'Agriculture et le PNR à propos des mesures agri-environnementales (MAE) et des contrats d'agriculture durable (CAD).

13. Correspondance datée du 22.04.2003.

14. Soit 6 sites sur les 7 plans de gestion en cours de réalisation dans la région, début 2005 (avant l'adoption de la loi DTR).

15. Situé en terrain accidenté, le long d'un cordon d'environ $20 \mathrm{kms}$ de long sur 300-400 m de large, ce site d'une superficie de 429 ha ne présente pas d'intérêts économiques majeurs. Il est composé à $60 \%$ de forêt (mélange de taillis et de futaie) et $40 \%$ de milieux ouverts conquis en partie par la friche. La propriété est à dominante privée (85\%). Les terrains publics sont en partie détenus par le Conseil général du Pas-de-Calais au titre des Espaces naturels sensibles et font l'objet d'un arrêté de protection de biotope; la fraction restante appartient à des collectivités locales et est classée au titre de réserve naturelle.

16. Organisme en charge de la gestion des espaces naturels sensibles du Conseil Général du Pas de Calais.

17. Si ce découpage par entité de gestion répondait aux exigences du CRPF et de la Chambre d'Agriculture et correspondait assez bien aux attentes d'Eden 62, il ne satisfaisait guère le Parc 
habitué à construire des partenariats et qui se trouvait, dans le cas présent, acculé sur une portion restreinte de son territoire.

18. Extrait de la convention cadre de coopération entre les opérateurs du site NPC33.

19. Soit un représentant de chaque structure, assisté d'un chargé de mission.

20. Extrait de la convention cadre de coopération entre les opérateurs du site NPC33.

21. Cette démarche volontariste est restée l'apanage des forestiers privés dans la région, à l'exception de la Chambre d'agriculture du Nord qui s'est, elle aussi (sans doute sous l'influence $\mathrm{du}$ (RPF), engagée à réaliser les inventaires sur quelques sites, tout en reconnaissant les difficultés de l'entreprise.

22. Extrait du rapport d'évaluation des docob des sites NPC 32, 33, 34, décembre 2003.

23. Celui-ci tend à faire de la conservation de la nature un principe légitime du «bien commun ».

\section{RÉSUMÉS}

Le recours à des procédures fondées sur la délibération dans le domaine de l'action publique a connu un essor important ces dernières décennies. Débattre, dialoguer, se concerter apparaissent désormais comme une nécessité, un impératif. Mais au-delà de ces déclarations d'intention, force est d'admettre que la concertation ne se décrète pas. Il ne suffit pas de rassembler des acteurs d'horizons divers autour d'une table pour qu'un véritable travail d'échange, de confrontation de points de vue et de connaissances s'élabore et débouche sur des accords. À partir de la mise en place d'une procédure de gestion concertée de la biodiversité instaurée dans le cadre de la directive Habitats en région Nord-Pas de Calais, nous voudrions rendre compte des difficultés et des obstacles auxquels se trouvent parfois confrontés ces modes d'action collective.

During the last decade, the use of procedure based on debate has known a great development in public action. Debating, discussing, consulting together are now recognized as something necessary and more, essential. Beyond this declaration of intent, it is necessary to admit that consulting together does not stand out. It is not enough to convene many stake-olders around a table to bring in a real debate, a real confrontation of different points of view and knowledge, and a least produce agreement. Through the implementation of a participatory approach to managing biodiversity used in the European Habitats directive, especially in the Nord-Pas de Calais Region, we shall examine difficulties and obstacles to implement this kind of collective action.

\section{INDEX}

Mots-clés : politiques publiques, concertation, Natura 2000, biodiversité, mesures de gestion, Nord-Pas de Calais

Keywords : public policy, participatory approach, biodiversity, management measure 


\section{AUTEUR}

\section{AGNÈS FORTIER}

Agnès Fortier, sociologue, est chargée de recherche à l'Institut National de la Recherche Agronomique (INRA), Unité Mona, Ivry-sur-Seine. Ses travaux portent sur la mise en œuvre de politiques publiques conduites au nom de la biodiversité (directive Habitats, Orientations régionales de gestion de la faune sauvage et des habitats (ORGFSH)). Elle vient de participer à la rédaction d'un ouvrage collectif intitulé « La construction du réseau Natura 2000 en France ». Elle est impliquée dans divers programmes : « Sociétés et cultures dans le développement durable » (ministère de la Recherche) et « Agriculture et développement durable » (ANR) et s'intéresse notamment à la construction sociale des normes et aux processus cognitifs qui les accompagnent. Courriel : fortier@ivry.inra.fr 